\title{
BMJ Open Preoperative bariatric surgery programme barriers facing Pacific patients in Auckland, New Zealand as perceived by health sector professionals: a qualitative study
}

Tamasin Taylor, ${ }^{1}$ Wendy Wrapson, ${ }^{1}$ Ofa Dewes, ${ }^{2,3}$ Nalei Taufa, ${ }^{4}$ Richard J Siegert ${ }^{1}$

To cite: Taylor T, Wrapson W, Dewes 0, et al. Preoperative bariatric surgery programme barriers facing Pacific patients in Auckland, New Zealand as perceived by health sector professionals: a qualitative study. BMJ Open 2019;9:e029525. doi:10.1136/ bmjopen-2019-029525

- Prepublication history and additional material for this paper are available online. To view these files, please visit the journal online (http://dx.doi org/10.1136/bmjopen-2019029525).

Received 30 January 2019 Revised 11 September 2019 Accepted 16 0ctober 2019

Check for updates

(C) Author(s) (or their employer(s)) 2019. Re-use permitted under CC BY-NC. No commercial re-use. See rights and permissions. Published by BMJ.

For numbered affiliations see end of article.

Correspondence to

Dr Tamasin Taylor;

tamasin.taylor@aut.ac.nz

\section{ABSTRACT}

Minority ethnic patient groups typically have the highest bariatric surgery preoperative attrition rates and lowest surgery utilisation worldwide. Eligible patients of Pacific Island ethnicity (Pacific patients) in New Zealand (NZ) follow this wider trend.

Objectives The present study explored structural barriers contributing to Pacific patients' disproportionately high preoperative attrition rates from publicly-funded bariatric surgery in Auckland, NZ.

Setting Publicly-funded bariatric surgery programmes based in the wider Auckland area, NZ.

Design Semi-structured interviews with health sector professionals $(n=21)$ were conducted.

Data were analysed using an inductive thematic approach.

Results Two primary themes were identified: (1)

Confidence negotiating the medical system, which included Emotional safety in clinical settings and Relating to non-Pacific health professionals and (2) Appropriate support to achieve preoperative goals, which included Cultural considerations, Practical support and Relating health information. Clinical environments and an under-representation of Pacific staff were considered to be barriers to developing emotional safety, trust and acceptance of the surgery process with patients and their families. Additionally, economic deprivation and lower health literacy impacted preoperative goals.

Conclusions Health professionals' accounts indicated that Pacific patients face substantial levels of disconnection in bariatric surgery programmes. Increasing representation of Pacific ethnicity by employing more Pacific health professionals in bariatric teams and finding novel solutions to implement preoperative programme components have the potential to reduce this disconnect. Addressing cultural competency of staff, increasing consultancy times and working in community settings may enable staff to better support Pacific patients and their families. Programme structures could be more accommodating to practical barriers of attending appointments, managing patients' preoperative health goals and improving patients' health literacy. Given that Pacific populations, and other patients from minority ethnic backgrounds living globally, also face high rates of obesity and barriers accessing bariatric surgery, our findings are likely to have broader applicability.
Strengths and limitations of this study

- Health sector professionals of Pacific ethnicity were able to contribute insights from both their professional experiences and their cultural knowledge.

- Using qualitative methodology, the present study extends and enriches previous quantitative research on preoperative barriers facing minority populations.

- It was not possible to determine exactly which structural barriers have the most impact on bariatric surgery attrition.

\section{INTRODUCTION}

Race and ethnicity persist in being significantly linked to quality of healthcare received, even after controlling for socioeconomic factors. ${ }^{1}$ Studies in the USA, Canada, Australia and New Zealand (NZ) indicate that minority ethnic patient groups have high population needs for bariatric surgery but are typically under-represented in surgery utilisation. ${ }^{2-6}$ Factors contributing to preoperative bariatric surgery attrition include: patients failing to meet programme requirements (such as weight loss requirements, increasing physical activity and attending mandatory appointments), psychiatric conditions, male gender, older age, higher Body Mass Index (BMI), insurance denials (in the USA) and higher substance use. ${ }^{7-12}$

Pacific people in NZ account for around $7.4 \%$ of the population and are made up of a diverse range of ethnic groups. ${ }^{13}$ People of Samoan ethnicity make up $48.7 \%$ of Pacific populations in NZ, followed by Cook Islands Māori (20.9\%), Tongan (20.4\%) and Niuean $(8.1 \%) .{ }^{13}$ Almost $70 \%$ of Pacific populations live in Auckland City. ${ }^{13}$ On average, Pacific populations have lower household incomes ${ }^{14}$ and higher rates of poverty ${ }^{15}$ compared with the general population. 
All Pacific cultures are traditionally highly collectivist, in that self-identity, goals, emotional well-being and material resources are an inseparable part of family, extended family and community groups. ${ }^{16-20}$ Many Pacific people share holistic and spiritual views of health that place importance on balanced relationships with others, and the environment. ${ }^{16}$ Additionally, around $83 \%$ of Pacific people identify as religious compared with around $47 \%$ of New Zealanders from other ethnic groups. ${ }^{20}$ In terms of physical health, Pacific populations in NZ have the highest obesity and related non-communicable diseases, including type 2 diabetes mellitus (T2DM) and hypertension. $^{2122}$

NZ is divided up into 21 District Health Boards (DHBs). These DHBs are independently responsible for the planning, management and provision of service funding of primary healthcare, including publicly funded bariatric surgery programmes. ${ }^{22}$ Surgery programme structures reflect that of the wider health system in NZ which is characterised as a 'medical model' of provision that focusses on treating the physical and biological aspects of specific diseases and conditions. ${ }^{22-24}$ This system has been criticised for discluding wider determinants of health such as socioeconomic factors. ${ }^{23}{ }^{24}$ As a result, equitable access to improved health is not shared across population groups. ${ }^{22}{ }^{25}$ Additionally, bias towards patients from indigenous and minority groups has been identified in such medical settings in both NZ and globally. ${ }^{25-27}$

Patients can be referred to a DHB bariatric surgery programme by a general practitioner (GP) or other health specialist (for example, a diabetes specialist). Acceptance criteria follows international guidelines (for example, those found in the 2016 International Federation for the Surgery of Obesity and Metabolic disorders (IFSO) guidelines). ${ }^{28}$ Patients are assessed on a case by case basis, however, requirements typically include a BMI $\geq 35 \mathrm{~kg} / \mathrm{m}^{2}$, a history of failed weight-loss attempts, and at least one obesity-related comorbidity known to improve consequent to surgery. Super-obese (BMI $>50 \mathrm{~kg} / \mathrm{m}^{2}$ ) patients may be declined if they are deemed as too high risk. Once accepted onto a programme, patients enter the 'preoperative' phase which involves attending compulsory appointments with the bariatric team. These appointments may be with dietitians, nurse specialists, surgeons and health psychologists. Some DHBs require patients to attend group support sessions and most programmes require patients to quit smoking. Patients are typically given a weight-loss target calculated at $10 \%$ of excess body weight above a BMI of $25 \mathrm{~kg} / \mathrm{m}^{2}$ and to follow a very low calorie diet for approximately 3 to 6 weeks before the surgery date.

Patients of Pacific ethnicity in NZ are the least likely to complete publicly-funded surgery ${ }^{6}$ despite having $^{6}$ the highest need for obesity treatment by population. An audit study at Auckland City Hospital reported that $73 \%$ of Pacific patients dropped out of the bariatric surgery preoperative programme compared with $39 \%$ of patients of NZ European ethnicity. 'Disengagement' from the programme was identified as the most salient factor related to attrition. This was defined as a patient failing to attend scheduled appointments, dropping out of the programme without giving a reason, or avoiding attempts to contact them. The authors noted that it was not possible to ascertain the reasons that led to Pacific patients disengaging from the service.

One study was able to elucidate a number of reasons behind the high Pacific patient attrition rates from primary healthcare and outpatient services in NZ. ${ }^{29}$ The authors found that it was particularly common for satisfactory patient-practitioner relationships to be lacking. Other barriers Pacific health consumers faced included transport problems, difficulties making appointments, patient anxiety and a lack of confidence in communicating with health professionals. Additionally, patients were often fearful of medical processes and had competing priorities that kept them from following through with treatment. Health professionals interpreted misunderstandings with their Pacific patients as a consequence of their patients' low health literacy, communication difficulties related to English being a second language and patients' resistance to lifestyle changes. While this study highlighted barriers in the primary-care sector, there is nevertheless a gap in explaining Pacific patients' preoperative attrition specific to bariatric surgery. This is a major limitation to planning effective countermeasures that could improve retention and decrease systemic bariatric surgery disparities.

Grounded in social constructionism, ${ }^{30} 31$ the present study aimed to explore health professionals' views on the barriers contributing to Pacific patients' disproportionately high preoperative attrition rates from publicly-funded bariatric surgery in Auckland, NZ. Social constructionism is an epistemological perspective that is concerned with how knowledge is 'constructed'. From a social constructionist perspective, people learn from each other through social interaction and together they create knowledge or shared understandings. The focus of this study, therefore, was on examining health professionals' understandings of Pacific patients' decision-making in going ahead with surgery and the factors that may contribute to withdrawal from a bariatric surgery programme.

\section{MATERIALS AND METHOD}

Semi-structured interviews were conducted to explore health sector professionals' experiences and perspectives from working with Pacific patients. Participants were eligible to be interviewed if they were: (a) Pacific or nonPacific health sector professionals who work directly with patients in a bariatric programme or in related community health services, or (b) Pacific health sector professionals who work with Pacific patient groups in hospitals or in community services. Professionals from both Pacific and non-Pacific ethnicities were recruited to offer viewpoints from different cultural lenses.

The interview guide was first constructed by two of the authors (TT and NT) based on the existing literature 
related to preoperative bariatric surgery attrition and barriers for Pacific patients in the healthcare system. Questions were then reviewed by the rest of the research team. The interview guide was designed to have the flexibility to follow divergent lines of enquiry and funnelling was employed to elicit both the respondent's general views as well as their response to more specific aspects of the topic (see online supplementary file 1).

Expert sampling was used to recruit participants from three DHBs that cover the wider Auckland area (namely, Counties Manukau, Auckland and Waitemata). The interviewer and first author (TT) is female, of NZ European and Pacific heritage and holds a PhD in Health Psychology. She was motivated to investigate the issue of obesity and bariatric surgery due to observing many of her own family members of Pacific heritage living with obesity and T2DM. The interviewer had met three of the participants previously. She had met one of the bariatric surgeons and the diabetes specialist while planning the research to discuss the feasibility and importance of conducting the study. Both specialists were concerned about the high programme attrition rates and wished to understand the barriers facing Pacific patients. The interviewer had met another of the participants, a Pacific community support worker, in a professional capacity in a previous role. None of these participants had any involvement in the study design or analysis.

From 32 study invitations sent to participants' work email addresses, 21 participants (14 female), of whom eight were of Pacific heritage, agreed to be interviewed (table 1) Transcripts were thematicised as the study

Table 1 Professional role and ethnicity of participants

Mixed

Role Pacific heritage* Non-Pacific

Pacific health/planning 2
and funding

Public health physician

$\begin{array}{lll}\text { Bariatric surgeon } & 1 & 3 \\ \text { Bariatric health } & & 1 \\ \text { psychologist } & & \end{array}$

$\begin{array}{ll}\text { Bariatric dietitian } & 3 \\ \text { Bariatric clinical nurse } & 3 \\ \text { specialist } & \\ \text { Pacific community health } 1 & \\ \text { worker }\end{array}$

Pacific health navigator 1

$\begin{array}{ll}\text { Diabetes specialist } & 1 \\ \text { GP liaison officer } & 1 \\ \text { GP } & 1\end{array}$

Registrar surgeon 1

Surgical bookings clerk 1

*Mixed heritage refers to participants who were part Pacific and part non-Pacific. progressed, and after interview 21, recruitment stopped. At this point, there was sufficient saturation of themes. Reasons for non-participation were not collected. The digitally-recorded interviews were undertaken at participants' workplaces or nearby locations and lasted from $40 \mathrm{~min}$ to 2 hours (average length: $90 \mathrm{~min}$ ). Participants were given a $\$ 40$ voucher in appreciation of their time. The interview recordings were transcribed verbatim; participants had 3 months to review their interview transcript and were free to clarify any information they had offered. To preserve participants' anonymity, professional roles are listed in table 1 , however they have not been attached to specific contributions in the findings.

\section{PATIENT AND PUBLIC INVOLVEMENT}

No patients involved.

\section{DATA ANALYSIS}

Data were analysed using an inductive thematic approach $^{32}$ focusing on clinical environments, treatment process and instances of personal interactions in participants' narratives that may suggest barriers for Pacific patients taking part in the bariatric surgery programmes. Inductive thematic analysis is a 'bottom-up' process using participant interview scripts as the data to systematically build themes. From a social constructionist perspective, how a patient, practitioner or society views medical treatment and the treatment process is formed through interactions and institutionalised practices situated within historical and sociocultural contexts. ${ }^{30}{ }^{31}$ In keeping with a social constructionist stance, which allows for people to create knowledge through social processes yet retain individual viewpoints, this approach enabled identification of converging and diverging instances across participants' interview dialogue that signalled socially or culturally constructed views of the barriers facing Pacific bariatric surgery patients.

The first (TT) and fourth (NT) authors independently read the scripts numerous times to achieve immersion in the data. NVivo V.11 $11^{33}$ was used to code and develop overarching themes and subthemes from the data that reoccurred throughout participants' narratives. TT and NT separately created thematic tables which were then compared and a main table of themes was agreed on. The themes were then discussed by the research team and a consensus reached on the final themes and subthemes.

Pacific and non-Pacific participant interview data were compared and were found to hold more similar than dissimilar perceptions of the barriers, experiences and interactions contributing to Pacific bariatric surgery patients' attrition rates. For the few themes where there were some nuances or additional explanation from Pacific health professionals' cultural perspectives, these are discussed in the findings. The Consolidated Criteria for Reporting Qualitative Research framework guided the reporting of the findings. ${ }^{34}$ 


\section{RESULTS}

Two primary themes were identified: (1) Confidence negotiating the medical system, which included Emotional safety in clinical settings and Relating to non-Pacific health professionals and (2) Appropriate support to achieve preoperative goals, which included Cultural considerations, Practical support and Relating health information.

\section{Theme 1: confidence negotiating the medical system}

Participants discussed the barriers facing their Pacific patients in relation to the hospitals' clinical settings. Such environments could be particularly challenging when it came to patients feeling a sense of emotional security and trust of medical processes.

\section{Subtheme 1: emotional safety in clinical settings}

Participants felt that Pacific patients were motivated to fulfil preoperative programme requirements; however, patients were often observed as being 'out of their depth' in the NZ medical system which was seen as difficult to negotiate without the right level of confidence.

I do see the health system as quite intimidating... when you see a Pacific Island patient in the waiting room, particularly if their English isn't very strong...I hear health professionals talk about Caucasian or New Zealanders have more of that confidence to negotiate and push, push their...what they need through, compared to Pacific people. (Non-Pacific Bariatric specialist)

Some participants referred to 'surgeon aura' and 'white coat syndrome' to indicate the effect surgeons had on Pacific patients who were seen as less confident or willing to ask questions about medical processes.

You try to encourage them to do so [to ask questions], but I guess Pacific culture is the doctor is someone that's special and you do what they say. You never question them... (Non-Pacific Bariatric specialist)

Body language was sometimes observed to be more withdrawn and disengaged in Pacific patients.

I definitely notice that they [Pacific patients] don't necessarily look at you. By western or European standards, that comes across as very withdrawn or possibly disinterested, to an extent. (Non-Pacific Bariatric specialist)

At two out of the three bariatric surgery programmes included in this study, patients are required to attend compulsory group support meetings. These meetings are considered important for patients to feel supported and to ask questions they may not have the confidence to ask in one-to-one consultations. Participants observed, however, that meetings were sometimes 'overtaken' by NZ European patients and they questioned whether Pacific patients were comfortable or confident in voicing their concerns in such a context.
I notice that a significant amount of Pacific people only listen. They don't really contribute in discussions, like it's really hard to know if they get the ideas or they don't understand or whether they do but they don't want to say. (Non-Pacific Bariatric specialist)

Some Pacific participants explained that, for many Pacific people, hospitals are institutions that symbolise disease, impending death and the prospect of dying on the operating table instead of in their own home. This was one reason suggested as to why some Pacific patients were intimidated by clinical environments.

...back in the Islands people don't go to the hospital unless they're really really sick and often they don't come out of hospital. I mean they do, but in a casket so there is a lot of fear around surgery and hospital services and stuff like that. (Pacific health professional)

Time was considered necessary to build patients' trust with health professionals and to view hospital spaces in a more positive light. Many participants commented on the particular importance of establishing emotional safety, understanding and familiarity. This could allay patients' fears around hospitals and medical processes as well as increase motivation. Participants acknowledged that the brief and infrequent appointments throughout the presurgery phase were seen as a format that undermined this process.

...we can have like only 20 min to talk to them. That is another barrier actually, 20 min to talk about major issues. You really can't solve the world in $20 \mathrm{~min}$.

(Non-Pacific Bariatric specialist)

Taking some bariatric services, such as group support meetings or appointments with dietitians and nurse specialists, out of the clinical environment and into the community was suggested. This could be a way to potentially lessen the perception that the preoperative process was a purely clinical process and to build patients' feelings of emotional safety.

We know they need more support, wider community engagement and we just can't do it. We don't have the resources to do it. If we continue to get more of those groups (Pacific patient groups), perhaps it'll be better off for those groups going to seminars and church groups, for them to feel more comfortable and more engaged. (Non-Pacific Bariatric specialist)

\section{Subtheme 2: relating to non-Pacific health professionals}

Some participants believed that Pacific patients could not easily relate to health professionals who, in general, represent highly educated, NZ European people the participants described as 'naturally' slim. They felt that patients may resist engaging with health professionals due to feeling ashamed, judged and misunderstood 
by them, particularly if a patient displayed low English proficiency, or was struggling to meet preoperative requirements.

...you have to understand that the people that are providing the care are people like me who are skinny, white, educated women who do not understand that fundamental culture, there is this disconnect straight away. (Non-Pacific Bariatric specialist)

When they see white and slim people, they think oh it's only white people that can do it. Like that's their build is naturally slim. "What do they know? They've never been big in their life...". It would be nice to have a Pacific Island nutritionist, or maybe [a nutritionist who] was big and has gone through and now does diet and exercise. (Non-Pacific health professional)

Both Pacific and non-Pacific participants noted that while NZ health professionals were competent and knowledgeable with their patients, there was sometimes a lack of emotional connection. This could hinder levels of motivation to follow through with the surgery for some Pacific patients.

I think a lot of the Palangi [NZ European] professionals do not connect emotionally. They connect intellectually, they have passed across the information and they think they've done the job. But not emotionally, which is important because it's part of their [Pacific patients'] motivation. (Pacific health professional)

Two Pacific participants illustrated how they believed they were better equipped than non-Pacific health professionals to assist Pacific patients navigate the preoperative stage, relating to them from a platform of implicit understanding.

When I talk to them and I know they are Pacific, their voices change, I really try and help them to make it easier for them. Sometimes when you speak to them you realise that they have broken English so I go down to their level... some Pacific people I have convinced them to go through with the surgery, I tell them how good it is for her or his own good. (Pacific health professional)

People are not just motivated by their knowledge. But actually, the motivation comes from what are their goals in life, what do they want to stay well for. A Pacific [person] can say that to another without sounding patronising. (Pacific Bariatric health professional)

\section{Theme 2: appropriate support needed to achieve preoperative goals}

The level of support available for Pacific patients to successfully traverse the bariatric surgery journey was discussed by participants. Three subthemes emerged from this discourse: Cultural considerations, Practical support and Relating health information.
Subtheme 1: cultural considerations

Participants identified that for Pacific patients, health professionals needed to establish relationships with not only the patient, but importantly, with patients' families if possible.

When you're managing Pacific patients, who are waiting for surgery, you have to build up a certain degree of trust. That trust doesn't just extend for patients, as it would for non-Pacific patients, but for Pacific it's unique in that it has to extend for their families as well. (Non-Pacific Bariatric specialist)

...they [Pacific patients] put more value on different things, like face to face stuff, one on one stuff, trying to establish relationships and maybe the service hasn't been resourced to do that stuff. (Non-Pacific Bariatric specialist)

A Pacific participant explained that for many Pacific patients the 'voice' of the family was often more highly respected than advice from health professionals, even if they were at risk of negative health consequences as a result. This illustrated how crucial it was to have the families on board in the patient's journey.

When you [the patient] don't have the resource and the family's voice is still stronger, it's hard to counter that. So, you just come and think I'd rather die and have a family. (Pacific health professional)

Providing Pacific patient advocates, who can support patients and their families through the treatment process, was suggested as a way the service may be able to build relationships with patients' families.

...you need a team, or an individual, or resources to be there to provide the time and effort for people [patients] to build resilience. You know, get the person thinking, despite my family feeling that way, I can do this. (Pacific health professional)

At the same time, connecting patients with advocates needed to be treated carefully due to some Pacific patients not wanting to be the subject of 'talk' in their community. This observation was made by both Pacific and nonPacific health professionals. The primarily medical focus that bariatric services are structured around was seen by many as problematic due to a singular focus on the physical body, diet and exercise.

...bariatric surgery doesn't fit into the surgical model of let's take the thing out and send you on. (NonPacific Bariatric specialist)

...you've got to see the person as a whole entity. You can't just see them as just physical and body side of things. (Non-Pacific Bariatric specialist)

Participants observed there were factors of higher importance for many Pacific patients than going through with surgery, such as the impact of treatment on their families, their ability to manage their responsibilities and 
their overall well-being. Two Pacific participants indicated that Pacific patients would be more likely to go through with surgery if the 'motivational' element was based on well-being and goals that were centred around being active for their family and in their community.

Pacific people have so many strengths that we should be playing up and really using... if you can focus on wellness as opposed to illness, or you might die, or you might get diabetes. But you know having more energy or being around for your grandparents and children and be able to play with them and you know being spiritually well, being able to help out in your community. (Pacific health professional)

Pacific people are very focussed on community and family and so they're really big motivators. (Pacific health professional)

A few professionals had completed Pacific cultural competency courses or Pacific nutrition courses. This gave them an awareness of Pacific cultural perspectives around health and an ability to discuss some traditional Pacific foods in their patients' diets. Additionally, they became more aware of various social situations that may compete with their patients' abilities to fulfil preoperative goals. Since completing a cultural competency course, one non-Pacific health participant had observed that her Pacific patients never discussed their traditional foods at group meetings compared with other ethnic minorities. This indicated they may not feel comfortable doing so in that environment. This particular participant made a point of discussing traditional foods with her Pacific patients during face-to-face consultations. Generally, however, health professionals' heavy clinic-workloads and the low overall rates of Pacific patients in the programmes meant that completing competency courses was not an organisational, or individual, priority.

\section{Subtheme 2: practical support}

The design of preoperative programmes is based on the assumption that every patient has an equal ability to meet preoperative goals. The majority of participants, however, acknowledged that Pacific patients needed higher preoperative support to implement lifestyle changes. This was partly because these patients were more likely to have higher economic and social pressures compared with non-Pacific patients.

You know, if you have the financial luxury to say; 'ok this now is going to pay off in the long term', that's great. But if you have a boss that's saying 'you were off work last week and if you take another day off you've got no more sick leave', because don't forget, they are using all their sick leave because they are coming to the hospital for appointments all the time for other things. (Non-Pacific Bariatric specialist)

Scheduled appointments did not generally cater for those who had competing family responsibilities or work demands, despite some staff making the effort to find the most convenient times for patients. The timing of the appointments during working hours was an issue if wanting to bring family along.

You've got an appointment at the hospital at 2pm. You want your husband or your wife or your children to come with you. They're all working. So it's enough that you are taking time out to go to the hospital, but they can't come with you. (Non-Pacific Bariatric specialist)

The access to and cost of transport, the distance to the hospital and parking costs (at one of the DHBs) were considered to be barriers for Pacific patients who might not be familiar with the medical system and unable to cover transport and other costs.

There are lots of disincentives blocking [Pacific] people from coming in such as parking prices, getting time off work, cost of parking and time and children care, all sorts of issues. (Non-Pacific Bariatric specialist)

Perhaps due to the required level of support not being received, Pacific patients had higher rates of not attending scheduled appointments without giving a reason. Staff have limited time available to follow-up on these patients who then became lost to the system if no direct or timely contact was made. Some participants suggested the use of online sessions and text messaging to help patients manage schedules and to find ways to relieve cost barriers associated with transport, parking or childcare.

\section{Subtheme 3: relating health information}

Participants pointed to health literacy as being notably lower among Pacific patients regarding dietary requirements. They were found to be more likely to diverge from specific dietary instructions despite health professionals believing the instructions they relayed were well understood.

She [the Pacific patient] seemed like she understood as she was repeating things back to me about what she should do. The day before her surgery...it turns out she had been eating the whole time with the Optifast protocol so hadn't followed anything so her surgery got cancelled. (Non-Pacific Bariatric specialist)

It was suggested that some Pacific patients could not envisage the future implications of remaining obese, particularly if they lived in environments where the average person was obese and yet remained active. This was more apparent among patients who reported feeling few symptoms, especially when they considered themselves generally healthy.

When you live in an environment where everyone has a weight issue or where they are big, that doesn't necessarily correlate to you as there is a problem. And if you don't think like it's a problem, then why are you going to look for a solution? (Non-Pacific Bariatric specialist) 
One challenge observed by health professionals was finding strategies to educate absent, or unsupportive, family members around why having surgery was a necessary treatment for their family member and why making lifestyle changes before surgery was important.

For those with limited English, translators were available to attend patient appointments but only on an as-requested basis. It was considered that Pacific patients may not be aware of this service; for example, one surgeon reported never having had a translator requested by any of his Pacific patients. Other participants had experienced that translators could make the patient-practitioner relationship less connected, and there were concerns around whether translators were relaying accurate information to patients. It was suggested that Pacific patients had the option of using any NZ-educated family members to translate resources and complete questionnaires. Pacific participants pointed out, however, that this could be culturally inappropriate or embarrassing for a patient; for example, if the family member was younger and they had to read out or request personal information.

\section{DISCUSSION}

The findings from this study help to illuminate the medical and social milieux in which surgical intervention for obesity is carried out in NZ. They reveal that interviewees viewed the health professional-patient interactions and institutional structures as contributing to the high attrition rate of Pacific patients in relation to bariatric surgery. The clinical environment and biomedical leanings of the preoperative programmes combined with an under-representation of Pacific staff were considered by health sector professionals to be key barriers for retaining Pacific bariatric patients, who were often observed to be relatively disempowered in such settings.

Patient-practitioner consultations did not incorporate relational and health belief values that were important in motivating many Pacific patients. In particular, the current study findings indicated that the practical decision-making side of health, illness and healing was a 'family affair' for many Pacific families and patients' family members needed to be involved in their healthcare planning. Participants' perspectives are supported by a review that found biomedical-based programmes aimed at changing health behaviour were shown to be ineffective, particularly for Pacific people. ${ }^{16}$ Of relevance to the present study, instructions by health professionals to Pacific patients to 'eat less' in the name of avoiding negative health consequences were found to be problematic when weight and food choices were affected by cultural norms and economic factors.

A recent review concluded that preparation for bariatric surgery has unique lifestyle change requirements compared with other surgeries that are arguably more suited to the biomedical model (eg, knee or eye surgery).$^{35}$ In particular, successful treatment outcomes require patients to make significant lifelong changes to eating and physical activity patterns that can have wideranging psychosocial effects. ${ }^{35}$ As such, preoperative bariatric surgery programmes may potentially be better suited to an alternative treatment paradigm that can work with these individual considerations while at the same time retaining a goal to prepare patients medically.

There were some important implications for clinical practice and policy. First, building practitioner-patient relationships that respect the need for increased time to develop trust and acceptance of the surgery process with patients and their families was a consistent theme across interviews and is an issue identified in previous literature. ${ }^{162936}$ Consideration should be given to cultural competency courses being mandatory to train health professionals in how to foster culturally-appropriate relationships with Pacific patients and their families. This may go some way to reducing the sense of anxiety and disempowerment that Pacific patients were observed to exhibit in clinical settings. Otherwise known as 'cultural responsiveness', developing cultural competencies has been recommended in previous reports as a priority to improve health for Pacific populations. ${ }^{37}$

Participants considered that the preoperative health behaviour change requirements to qualify for surgery were particularly demanding for Pacific patients who may live in more challenging socioeconomic situations compared with patients of other ethnicities. This is reflected in the wider literature investigating socioeconomic barriers to health in $\mathrm{NZ}^{19-2238}$ and bariatric surgery utilisation in the UK, the USA, Canada, Australia and Sweden. $.^{2-5} 39-41$ Such patients are exposed to more obeseogenic factors and are less able to offset risk factors given higher financial pressures and lower education levels. However, programmes were not resourced sufficiently to support Pacific patients who may be struggling with such challenges. It is clear that more funding support should be considered at the policy level for patients affected by economic deprivation.

In terms of health literacy, there is a need for bariatric surgery practices to consider ways to support Pacific patients who are not following through with dietary advice and those who are unable to envisage the implications of remaining obese. The wider literature indicates that how a patient understands and responds to health information needs to be recognised in the context of culture. ${ }^{42}$ There may be differing styles of communication, in the meanings of words and gestures and what can be discussed regarding the body, health and illness. ${ }^{42}$ In the present study there were various accounts of Pacific patients exhibiting styles of communication that differed from patients of other ethnicities and instances of miscommunication between patients and practitioners. Finding culturally appropriate ways of communicating with Pacific patients could be reviewed as part of future research and implemented at clinical and policy levels. To increase cultural competency of the service and achieve higher retention rates, it would be worthwhile exploring the feasibility of a Pacific-led, culturally informed prebariatric surgery support programme. 
One of the main limitations in the present study is that the number of non-Pacific participants outnumbered the Pacific/mixed heritage participants, and there was almost no overlap in professional roles. This may potentially contribute to a bias in terms of perspectives. In particular, there was a lack of Pacific participants who worked in the surgery teams. This unequal distribution of ethnicities employed in these medical roles is a direct reflection of the demographics of the medical profession in NZ. ${ }^{43}$ There is a paucity of Pacific bariatric surgeons, nurse specialists, dietitians and health psychologists working in these three wider Auckland DHBs. Training of Pacific health professionals is recommended given the lack of this ethnic representation.

A strength of the study is that the participants have had a vast array of differing professional contacts and experiences with many Pacific patients. On the other hand, it is important to also interview patients who have had direct experiences of presurgery programmes to explore and add to the themes elicited from the present sample. As such, a subsequent study by the authors will explore Pacific bariatric surgery patients' perspectives enabling a comparison of practitioners' with patients' experiences.

\section{CONCLUSION}

This study highlighted structural areas of current bariatric surgery programmes in Auckland, NZ that may be failing our Pacific patients. While this patient group is a small subset of patients overall, they come from a population that experiences some of the highest levels of metabolic-related conditions worldwide. The findings and recommendations have the potential to be generalised to other ethnic minority groups who may also face barriers in accessing bariatric surgeries due to structural and sociocultural barriers. For example, the indigenous Māori population in NZ also faces significant disparities in accessing bariatric surgery treatments, higher rates of obesity-related diseases and lower socioeconomic status compared with NZ European health consumers. ${ }^{6}$ Key areas in bariatric services were identified that may be amenable to structural change. Specifically, preoperative processes preparing patients for surgery could be reconfigured to allow for increased emotional safety, higher cultural awareness and more effective management of the practical needs of patients; patients who potentially have much to gain from this life preserving treatment.

\section{Author affiliations}

${ }^{1}$ Faculty of Heath and Environmental Sciences, Auckland University of Technology, Auckland, New Zealand

${ }^{2}$ Maurice Wilkins Centre for Molecular Biodiscovery, University of Auckland, Auckland, New Zealand

${ }^{3}$ Department of Molecular Medicine and Pathology, University of Auckland, Auckland, New Zealand

${ }^{4}$ School of Population Health, University of Auckland, Auckland, New Zealand

Contributors Study conception and design overseen by all authors (TT, WW, OD, NT, RJS). Draft written by TT and WW. Draft critically revised by all authors (TT, WW,
OD, NT, RJS). Thematic analysis by TT and NT. Theme checks by all authors. All authors agree to be accountable for all aspects of the work and agreed on the final manuscript.

Funding The research was conducted during the tenure of a Pacific Health Research Postdoctoral Fellowship of the Health Research Council of New Zealand awarded to the first author (HRC 17/472). The funders had no role in data collection, interpretation and reporting.

Competing interests The submitting author (Dr Tamasin Taylor: author 1) and the other four contributing authors (author 2: Dr Wendy Wrapson, author 3: Dr Ofa Dewes, author 4: Miss Nalei Taufa, author 5: Professor Richard John Siegert) declare they have no affiliations with or involvement in any organsation or entity with any financial interest (such as honoraria; educational grants; participation in speakers' bureaus; membership, employment, consultancies, stock ownership, or other equity interest; and expert testimony or patent-licensing arrangements), or non-financial interest (such as personal or professional relationships, affiliations, knowledge or beliefs) in the subject matter or materials discussed in this manuscript.

\section{Patient consent for publication Not required.}

Ethics approval Ethics approval was granted by the Health and Disability Ethics Committee and by Auckland University of Technology Ethics Committee. Research locality approval from all participating DHBs was obtained.

Provenance and peer review Not commissioned; externally peer reviewed. Data availability statement № data are available.

Open access This is an open access article distributed in accordance with the Creative Commons Attribution Non Commercial (CC BY-NC 4.0) license, which permits others to distribute, remix, adapt, build upon this work non-commercially, and license their derivative works on different terms, provided the original work is properly cited, appropriate credit is given, any changes made indicated, and the use is non-commercial. See: http://creativecommons.org/licenses/by-nc/4.0/.

\section{REFERENCES}

1 Chapman EN, Kaatz A, Carnes M. Physicians and implicit bias: how doctors may unwittingly perpetuate health care disparities. J Gen Intern Med 2013;28:1504-10.

2 Sudan R, Winegar D, Thomas S, et al. Influence of ethnicity on the efficacy and utilization of bariatric surgery in the USA. J Gastrointest Surg 2014;18:130-6.

3 Wallace AE, Young-Xu Y, Hartley D, et al. Racial, socioeconomic, and rural-urban disparities in obesity-related bariatric surgery. Obes Surg 2010;20:1354-60.

4 Stanford FC, Jones DB, Schneider BE, et al. Patient race and the likelihood of undergoing bariatric surgery among patients seeking surgery. Surg Endosc 2015;29:2794-9.

5 Bhogal SK, Reddigan JI, Rotstein OD, et al. Inequity to the utilization of bariatric surgery: a systematic review and meta-analysis. Obes Surg 2015;25:888-99.

6 Rahiri J-L, Lauti M, Harwood M, et al. Ethnic disparities in rates of publicly funded bariatric surgery in New Zealand (2009-2014). ANZ J Surg 2018;88:E366-9.

7 Yang K, Zhang B, Kastanias P, et al. Factors leading to Self-Removal from the bariatric surgery program after attending the orientation session. Obes Surg 2017;27:102-9.

8 Taylor T, Wang Y, Rogerson W, et al. Attrition after acceptance onto a publicly funded bariatric surgery program. Obes Surg 2018;28:2500-7.

9 Sadhasivam S, Larson CJ, Lambert PJ, et al. Refusals, denials, and patient choice: reasons prospective patients do not undergo bariatric surgery. Surg Obes Relat Dis 2007;3:531-5.

10. Diamant A, Milner J, Cleghorn M, et al. Analysis of patient attrition in a publicly funded bariatric surgery program. J Am Coll Surg 2014;219:1047-55.

11 Pitzul KB, Jackson T, Crawford S, et al. Understanding disposition after referral for bariatric surgery: when and why patients referred do not undergo surgery. Obes Surg 2014;24:134-40.

12. Merrell J, Ashton K, Windover A, et al. Psychological risk may influence drop-out prior to bariatric surgery. Surg Obes Relat Dis 2012;8:463-9.

13. Pacific people in NZ. Ministry for Pacific peoples. Available: http:// www.mpp.govt.nz/pacific-people-in-nz [Accessed 7 Dec 2018].

14. Perry B. Household incomes in New Zealand: trends in indicators of inequality and hardship 1982 to 2013., 2014. Ministry of social 
development. Available: https://www.msd.govt.nz/about-msd-andour-work/publications-resources/monitoring/household-incomes/ [Accessed 13 Aug 2018].

15. Pasifika Futures. Pasifika people in New Zealand, 2015. Available: http://pasifikafutures.co.nz/wp-content/uploads/2015/06/PF_ HowAreWeDoing-RD2-WEB2.pdf [Accessed 20 Oct 2019].

16 Capstick S, Norris P, Sopoaga F, et al. Relationships between health and culture in Polynesia - a review. Soc Sci Med 2009;68:1341-8.

17 Podsiadlowski A. Collectivist Value Orientations among Four Ethnic Groups: Collectivism in the New Zealand Context [Internet]. New Zealand Journal of Psychology 2011;40.

18. Superu. Families: universal functions, culturally diverse values, 2017 Social policy evaluation and research unit. Available: https://thehub. sia.govt.nz/assets/Uploads/Families-culturally-diverse-AAG-190719. pdf [Accessed 7 Aug 2018].

19. Tukuitonga C. Pacific island health. Te Ara - the Encyclopedia of New Zealand. Available: https://teara.govt.nz/en/pacific-island-health/print [Accessed 7 Dec 2018].

20. 2013 census QuickStats about culture and identity. Stats NZ. Available: http://archive.stats.govt.nz/Census/2013-census/profileand-summary-reports/quickstats-culture-identity/pacific-peoples. aspx [Accessed 28 Mar 2018].

21 Utter J, Denny S, Teevale T, et al. Prevalence and recent trends in overweight, obesity, and severe obesity among New Zealand adolescents. Child Obes 2015;11:585-9.

22 Sheridan NF, Kenealy TW, Connolly MJ, et al. Health equity in the New Zealand health care system: a national survey. Int $J$ Equity Health 2011;10:45.

23. Fuller J. The new medical model: a renewed challenge for biomedicine.. CMAJ 2017;189:E640-1.

24. Trends in Service Design and New Models of Care - A Review, 2010. Ministry of health. Available: http://www.moh.govt.nz/NoteBook/ nbbooks.nsf/0/43C4C9001223BCBDCC257B10007AC681/\$file/ trends-service-design-new-models-care-jul2010.pdf [Accessed 10 Dec 2018]

25 Cormack D, Harris R, Stanley J, et al. Ethnic bias amongst medical students in Aotearoa/New Zealand: findings from the bias and decision making in medicine (BDMM) study. PLoS One 2018;13:e0201168.

26 van Ryn M, Saha S. Exploring unconscious bias in disparities research and medical education. JAMA 2011;306:995-6.

27. Nelson A. Unequal treatment: confronting racial and ethnic disparities in health care. J Natl Med Assoc 2002;94:666.

28 De Luca M, Angrisani L, Himpens J, et al. Indications for surgery for obesity and weight-related diseases: position statements from the
International Federation for the surgery of obesity and metabolic disorders (IFSO). Obes Surg 2016;26:1659-96.

29 Southwick M, Kenealy T, Ryan D. Primary care for Pacific people: a Pacific and health systems approach report to the health research Council and the Ministry of health, 2012. Available: http://www. health.govt.nz/publication/primary-care-pacific-peoplepacific-andhealth-systems-approach [Accessed 2 Apr 2018].

30 Crotty M. The Foundations of Social Research. Crows Nest, NSW, Australia: Allen \& Unwin, 1998.

31 Young RA, Collin A. Introduction: Constructivism and social constructionism in the career field. J Vocat Behav 2004;64:373-88.

32 Braun V, Clarke V. Using thematic analysis in psychology. Qual Res Psychol 2006;3:77-101.

33 Edhlund B, McDougall A. Nvivo 11 essentials 2017.

34 Tong A, Sainsbury P, Craig J. Consolidated criteria for reporting qualitative research (COREQ): a 32-item checklist for interviews and focus groups. Int J Qual Health Care 2007;19:349-57.

35 Sogg S, Friedman KE. Getting off on the right foot: the many roles of the psychosocial evaluation in the bariatric surgery practice. Eur. Eat. Disorders Rev. 2015;23:451-6.

36. Mauri Ora Associates. Best health outcomes for Pacific peoples: Pactice implications [Internet]., 2010. Medical Councilof New Zealand. Available: https://www.mcnz.org.nz/assets/News-andPublications/Statements/Best-health-outcomes-for-Pacific-Peoples. pdf [Accessed cited 2018 May 31].

37 Tiatia J. Pacific cultural competencies: a literature review. Wellington: Ministry of health, 2008.

38 Quirke T, Edwards D, Brewerton M, et al. Ministry of Pacific island affairs. health and Pacific peoples in New Zealand Pacific progress statistics New Zealand 2011.

39 Bhanderi S, Alam M, Matthews JH, et al. Influence of social deprivation on provision of bariatric surgery: 10-year comparative ecological study between two UK specialist centres. BMJ Open 2017;7:e015453.

40 Zhang JC, Tomlinson G, Wnuk S, et al. Disparities in receipt of bariatric surgery in Canada. Med Care 2019;57:723-7.

41 Memarian E, Calling S, Sundquist K, et al. Sociodemographic differences and time trends of bariatric surgery in Sweden 19902010. Obes Surg 2014;24:2109-16.

42 Nielsen-Bohlman L, Panzer AM, Kindig DA. Health literacy: a prescription to end confusion. Washington, D.C: The National Academies Press, 2004. http://nap.edu/10883

43. District health board employed workforce Quarterly report 1 April to 30 June 2018, 2018. Strategic workforce services, tas. Available: https://tas.health.nz/ [Accessed 1 Apr 2018]. 